\title{
La elaboración de política pública en los países periféricos y su diálogo con los organismos internacionales y el conocimiento especializado. Notas para su discusión desde el caso uruguayo
}

The elaboration of public policy in peripheral countries and their dialogue with international organizations and the specialized knowledge. Notes for the discussion from the Uruguayan case

\author{
YoAna PaOla CARballo PERDOMO*
}

\begin{abstract}
8003
RESUMEN - El artículo plantea notas sobre los procesos de elaboración de política pública en los países periféricos centrando la atención particularmente por un lado, en la participación de los organismos internacionales mediante lo que se denomina la asistencia internacional para el desarrollo, fenómeno que emerge luego de la segunda posguerra y se extiende hasta nuestros días; y por otro lado, en el papel relevante y sustantivo que el conocimiento especializado adquiere en los proceso de elaboración de política pública, donde las ciencias sociales ocupan un lugar destacado. De esta manera, trabajamos en este debate a partir del supuesto que la configuración de procesos de política pública significativos para los países periféricos se explica por una trama de relaciones entre actores nacionales e internacionales que lejos de ser lineal y simple es compleja y dinámica. Como tratamiento ilustrativo del debate esbozaremos algunas observaciones sobre el caso uruguayo y el proceso de reforma en el esquema de protección social en los últimos diez años mediante la implementación el Plan de Equidad Social (PES) a partir del 2008.**

Palabras clave - Política pública. Organismos internacionales. Conocimiento especializado.
\end{abstract}

ABSTRACT - The article presents notes on the processes of elaboration of public policy in peripheral countries, focusing particularly on the one hand on the participation of international organizations through what is called international assistance for development, a phenomenon that emerged after the second postwar period and extends to our days; Andon the other hand in the relevant and substantive role that specialized knowledge acquires in the process of developing public policy, where social sciences occupy a prominent place. In this way, we work in this debate based on the assumption that the configuration of significant public policy processes for peripheral countries is explained by a network of relationships between national and international actors that, far from being linear and simple, is complex and dynamic. As illustrative treatment of the debate, we will outline some observations on the Uruguayan case and the reform process in the social protection scheme in the last ten years through the implementation of the Social Equity Plan (PES) as of 2008.***

Keywords - Public policy. International organizations. Specialized knowledge.

\footnotetext{
* Doctoranda en Ciencias Sociales con especialización en Trabajo Social, Departamento de Trabajo Social y Magister en Trabajo Social, Departamento de Trabajo Social, Facultad de Ciencias Sociales, Universidad de la República, Uruguay. Docente e investigadora del Departamento de Trabajo Social, Facultad de Ciencias Sociales, Universidad de la República, Uruguay. E-mail: yoana.carballo@cienciassociales.edu.uy.

** Estas notas reflexivas se inscriben en las primeras exploraciones de nuestra investigación doctoral en curso, en la misma abordamos la participación de los organismos internacionales y el conocimiento especializado en la configuración de política socio asistencial de Uruguay entre el 2005 y 2015 en el marco del PES.

*** These reflexive notes are inscribed in the first explorations of our doctoral research in progress, in which we address the participation of international organizations and specialized knowledge in the configuration of social assistance policy in Uruguay between 2005 and 2015 within the framework of the PES. Presentado: julio/2017. Aprobado: septiembre/2018.
} 
a emergencia y progresiva expansión de la asistencia internacional para el desarrollo ha devenido en un fenómeno sustantivo para comprender y explicar la configuración de las políticas públicas en los

países periféricos. Si bien en un análisis general encontramos tendencias y parámetros que hacen un común denominador en todos los países de la región, existen y deben considerarse con especial atención, condiciones coyunturales y características propias de cada país en los procesos de elaboración de política pública, que, a su vez, varían de acuerdo con el sector de política analizando.

Esta consideración, por tanto, constituye además de un supuesto para esta presentación una pista metodológica para reflexionar de forma rigurosa sobre los procesos socio políticos que caracterizan a América Latina y las particularidades según el país y el sector de política. En sintonía con ello, el caso aquí puesto en debate, el proceso de reforma del esquema de protección social en Uruguay en los últimos diez años tiene como propósito mostrar particularidades propias, pero también ofrecer notas reflexivas que trascienden el caso y otorguen pistas para pensar en otras realidades, así como en la compleja dinámica que el mundo de las relaciones internacionales tiene actualmente, cuyo significado no es ni igual ni homogéneo para todos los países y regiones.

\section{Emergencia y expansión de la asistencia internacional para el desarrollo de los países periféricos}

En los países periféricos hacia finales del siglo XIX adquieren relevancia sustantiva en los procesos de gestión estatal distintas expresiones de la vinculación entre determinadas formas de conocimiento y su institucionalización, por un lado, y la formación de élites estatales expertas y el Estado por el otro. Acontecen dos procesos en simultaneo, por una parte, se generan requerimientos de saberes $y$ conocimientos específicos por parte del Estado para su legitimación cuya fuente encontró en las nacientes ciencias sociales modernas, al tiempo que, y, por otra parte, el Estado se constituye en un actor relevante para la consolidación de éstas (PLOTKIN; ZIMMERMANN, 2011).

Hacia finales de la Segunda Guerra Mundial con el surgimiento de un nuevo orden internacional ${ }^{1}$ emerge un elemento que caracteriza y explica hasta nuestros días parte de la dinámica que asume el orden internacional, la asistencia internacional para el desarrollo, cuya relevancia radica en el particular papel que ésta ha desempeñado en el proceso de cooperación de los países receptores del sistema internacional (MONTÚFAR, 2004).

No obstante, como bien recuerda Ornellas Mauriel (2008) en los primeros años del BM sus preocupaciones no estaban vinculadas a la pobreza, pues en sus primeras 26 memorias anuales el tópico pobreza raramente aparece, y cuando se hace presente su mención es rápidamente trabajada y asociada a la pobreza rural. Lo que evidencia el cambio de perspectiva de estes organismos en el transcurso de la historia, ya que, a su atención en aspectos financieros suma un repertorio de preocupaciones por cuestiones sociales, a saber, "Esse longo período de silêncio contrasta com os últimos anos, nos quais afirmações de compromisso social e redução da pobreza aparecem como marcas registradas" (p.23).

Existe una vasta literatura propositiva (FRERES, 2010; FRERES; MARTíNEZ; ANGULO, 2010; AUCI, 2011) producida desde espacios académicos, gubernamentales así como desde los mismos organismos internacionales ${ }^{2}$ que como señala Montúfar (2004), constituyen interpretaciones que comprenden y explican este fenómeno como geopolítico, tecnocrático o humanitario y que no profundizan en: i- despejar los motivos políticos, económicos y sociales que han impulsado y modelado este mecanismo de asistencia para el desarrollo de países periféricos; ii- la incidencia de la asistencia internacional para el desarrollo en la trayectoria de las políticas públicas de los países periféricos, en otras palabras, su incidencia en el proceso de "modernización" de las regiones y países asumidos como no desarrollados del planeta.

En igual sentido Martínez Franzoni (2006) entiende que los procesos de reforma de las políticas sectoriales en los países latinoamericanos, más allá de las macroeconómicas que han sido las más estudiadas, y la participación de los organismos internacionales (que la autora denomina como 
Instituciones Financieras Internacionales -IFIs-) ha sido poco explorado. Para la autora la relación entre los organismos internacioanles y las reformas sectoriales en cada país es una relación compleja, por un lado porque "(...) la influencia de estas agencias no necesariamente se ejerce de un modo tan directo y categórico como puede y suele hacerlo en el terreno de las políticas macroeconómicas", y por otro lado porque "(...) muy a menudo, las IFIs no tienen recomendaciones para las políticas sectoriales tan ortodoxas, estructuradas y estables como las que han elaborado en el plano de las políticas económicas clásicas" (p.128).

Entre los años 1950 y 1960 los organismos internacionales motivados por impulsar el desarrollo en los países periféricos centran sus acciones en inversiones en infraestructura y en asistencia financiera, ya que la pobreza de éstos representaba un peligro para el orden económico mundial que comenzaba a ser hegemonizado por Estado Unidos (MURILLO, 2006). En los inicios, los organismos internacionales en el conjunto de reformas impulsadas consideraron la reforma social como un elemento secundario y adicional a los objetivos macroeconómicos por ello proponen de forma general la misma sugerencia privatizadora y descentralizadora para el sector público en su conjunto.

Posteriormente en los 70 y 80, en particular el BM, si bien su preocupación central son las políticas financieras, a partir de la crisis de la deuda externa de varios países de la región en los 80 , al repertorio de preocupaciones por las políticas financieras y macroeconómicas se suma una concepción macroeconómica de la política social, a partir de lo cual pasan hacer objeto de particular atención las políticas sectoriales de los países (DE ARMA; GARCÉ, 2004; MARTíNEZ FRANZONI, 2006). En este momento la política del BM orienta sus sugerencias en principios de disciplina fiscal, en la reorientación del gasto público y reducción del gasto social, la liberalización financiera y comercial, las privatizaciones, la promoción de la inversión extranjera directa, el diseño de políticas sociales, las reformas educativas y transformaciones políticoinstitucionales entre otras, estas medidas acompañaban y hacían parte de la financiación brindada para reorientar la política económica de los países pobres (MURILLO, 2006).

Entre 1980 y 1990, principalmente el BM y el Banco Interamericano de desarrollo (BID), proponen un conjunto de reformas para "producir un disciplinamiento de las burocracias estatales" 3 que respondía a la necesidad de adaptarse estratégicamente a los cambios que el orden económico internacional establecía, así entonces, se comienza a forjar una burocracia en torno a estos organismos a modo de avanzar en el "disciplinamiento de las burocracias estatales" mediante programas de estabilización y programas de ajuste estructural de los estados. Estos programas tenían por objetivo primordial el cobro de la deuda externa que habían contraído en los 70 gran parte de los países subdesarrollados y la creación de condiciones propicias para los intereses de las transnacionales y el capital financiero. En este proceso de asistencia se preveía la existencia de distintos "círculos de participación" a manera de ir tornando una "relación informal en una relación oficial e institucional" que involucrara e hiciera participe a toda la burocracia de los estados. Es aquí donde se recupera como relevante el "papel del experto" con la finalidad que su lenguaje y códigos estandarizados sobre ciertos temas atenuara posibles dificultades enraizadas en "ciertos nacionalismos" e impulsara por tanto tratamientos comunes en distintos países (CORBALÁN, 2004, p.7-12).

Si bien es posible afirmar que a lo largo de la historia de la asistencia internacional para el desarrollo los intercambios se han establecido sobre un conjunto de principios, normas y reglas de procedimiento y organización, éstos se han organizado y operado con cierta flexibilidad según las coyunturas socio políticas, no obstante, ello, es posible afirmar que han emergido:

(...) procesos sostenidos de reforma estatal y convergencia de políticas públicas, función íntimamente conectada con la institucionalización de intercambios permanentes, normados y reglados (...) los países receptores adquirieron el "derecho" de recibir dichos flujos de asistencia, pero sobre la base de promover los cambios requeridos a nivel de sus estructuras políticas, económicas y sociales. (MONTÚFAR, 2004, p. 249) 
Existen varias producciones, aunque con matices en sus valoraciones sobre este proceso de asistencia internacional (MURILLO, 2006; MARTÍNEZ FRANZONI, 2006; CAMOU, 1997; CANZANI; CANCELA, 2015; DE ARMA y GARCÉ, 2004; CORBALÁN, 2004; ORNELLAS MAURIEL, 2008) que destacan por una parte, el papel relevante del conocimiento especializado en la estrategia de los organismos internacionales y por otra parte, una comprensión de este proceso de asistencia que trasciende lo financiero e involucra los aspectos económicos, políticos y culturales de los países.

En coherencia con estas afirmaciones, advierte Murillo (2006) que en esta creciente financiación y asistencia que estimula el BM como agencia financiera para el desarrollo de los países pobres se ha establecido un elemento relevante para el diseño de las políticas asociado a "la recopilación y análisis de datos sobre la pobreza", lo que está vinculado y en sintonía con la emergencia del paradigma sociotécnico que requirió de un elemento clave para su sustento: el conocimiento ${ }^{4}$. La autora identifica que fue en esta coyuntura que la investigación científica se tornó en elemento clave del nuevo paradigma y donde particularmente las ciencias sociales adquieren un papel relevante en tanto se vuelve fundamental el conocimiento de las poblaciones.

En la misma línea Martínez Franzoni (2006) recuerda que debe considerarse la importancia que adquiere el papel del conocimiento especializado en las reformas más allá de la fuerza de imposición que caracteriza ciertas agendas impulsadas por los organismos internacionales, ya que, “(...) no siempre la imposición de políticas es el principal objetivo de las IFIs sino que, muchas veces, su principal objetivo es la elaboración de dichas políticas mediante, precisamente, la ejecución de reformas domésticas", en otras palabras, el principal objetivo también pasa por la generación de aprendizajes y conocimientos (p.604).

Por tanto, no se trata de minimizar la incidencia de los organismos internacionales en los procesos de reforma de política pública sino de llamar la atención acerca del análisis que no es simple, sino que responde a una trama de relaciones entre diversos actores (políticos, técnicos-burócratas, expertos) así como a la coyuntura del momento (nacional e internacional) (DE ARMAS; GARCÉ, 2004). Camou (1997) también recuerda que no es una relación lineal la que explica el vínculo entre los organismos internacionales y las políticas públicas de distintos países, sino que es un proceso en el cual se hacen presente los intereses de los organismos internacionales, el propio Estado y agentes económicos, entre otros, cuya generalidad radica en que su lectura se orienta por principios de políticas públicas derivados de modelos y teorías económicas.

De este modo, el papel que fue adquiriendo el conocimiento especializado permitió la emergencia de un proceso de tecnificación de la gestión pública en general, y de la pobreza en particular, que colocó como objeto de estudio, el conocimiento de las poblaciones, de los procesos de trabajo, de la gestión y administración de los datos, de los nuevos riesgos sociales y de las estrategias para enfrentarlos. Y es en este sentido que la tecnificación de los procedimientos de gestión y el desarrollo de saberes sobre las características y comportamientos de los individuos se vuelven problemas de estudio para los cuadros burocráticos estatales, pues en gran medida la generación de instrumentos para abordar la nueva cuestión social mediante la "atención técnica", representa la posibilidad superadora del empobrecimiento. Este planteo recuerda lo señalado por Castel (2004) sobre la construcción y expansión de políticas que permiten tratar como "técnicos" problemas que esencialmente son "políticos", como es la producción y reproducción estructural de la pobreza y desigualdad.

La semántica discursiva de los organismos internacionales se caracteriza por la exposición de categorías interpretativas que explican la cuestión social y la pobreza como fenómenos manifiestos de la desigualdad social en tanto circunstancia inherente a la condición humana, en consecuencia, la igualdad es reemplazada por la idea de equidad, la cual admite niveles de desigualdad social. Es en estos términos que el BM propone hacer frente a la desigualdad social, mediante una táctica discursiva cuya visión sobre la pobreza se inspira en los desarrollos sobre la "teoría del capital humano" (MURILLO, 2006, p.55). Esta lectura hegemónica refuerza una representación y explicación de la pobreza como resultado, principalmente, de déficits individuales y familiares en la acumulación de "capital humano" 5 . (ÁLVAREZ LEGUIZAMÓN, 2011) 
De esta forma, los marcos cognitivos de referencia para la política asistencial se vuelven compresibles a partir de la "teoría del capital humano" cuyos desarrollos datan de la década de $1960^{6}$.

En el transcurso de los 80 tanto el BM como el BID expandieron sus departamentos especializados en política social, expertos y publicaciones en el área, y a mediados de los 90 ya tenían amplias capacidades instaladas para el diseño, financiamiento y asistencia técnica en materia de reforma social. Esta actitud frente a la generación de conocimiento ha llevado, fundamentalmente al BM, a constituirse como un organismo financiero, pero además a ser un referente en la teoría económica, social y en los estudios sobre el desarrollo. Desde la creación de grupos temáticos al desarrollo de bases de datos entre otras herramientas de análisis, permiten al BM generar, compartir y aplicar registros de información sobre los problemas que entiende merecen ser investigados en la región, las teorías a ser utilizadas para explicarlos, las hipótesis a ser trabajadas, las metodologías a ser implementadas, el estilo y el lenguaje, así como los términos y categorías "políticamente correctas" a ser empleadas (BORÓN, 2006; MURILLO, 2006; MATO, 2007; MARTÍNEZ FRANZONI, 2006).

\section{Aproximaciones al caso uruguayo}

Entrado el siglo XXI se coloca nuevamente el tema de la reforma social en Uruguay y la redefinición de la agenda social no sólo encuentra explicación en el contexto nacional, sino que remite también al conjunto de preocupaciones que los organismos internacionales visualizan ante los efectos sociales regresivos de las medidas de ajuste implementadas en los 90 con las reformas de "segunda generación".

En el 2002 el país vivió un escenario dramático generado por una crisis económica cuyos efectos sociales fueron profundamente terribles y se sumaron a una tendencia regresiva en los indicadores sociales. En este contexto de crisis asume el gobierno en 2005, por primera vez en la historia del país, un partido identificado con el espectro ideológico de izquierda, el Frente Amplio (FA).

El nuevo gobierno inauguró su gestión en el área social con tres medidas significativas en materia de bienestar: (i) la recreación de los Consejos de Salarios; (ii) el lanzamiento de un Plan de Atención Nacional a la Emergencia Social (PANES) proyectado para funcionar los dos primeros años y como respuesta inmediata a la situación profundamente crítica que padecía un sector importante de la población a raíz de la reciente crisis; y (iii) la creación de un organismo coordinador en este campo, el Ministerio de Desarrollo Social (MIIDES) (MIDAGLIA, 2008, p.101-102).

Posteriormente al PANES y a partir de 2008, se lleva adelante una iniciativa de Reforma Social que se plasma en el Plan de Equidad Social (PES), que recoge líneas de cambio y continuidad de su antecesor con el propósito de materializar y profundizar “(...) la estrategia de superación de la pobreza y reducción de la desigualdad social como condición de un país integrado e incluyente" (PLAN DE EQUIDAD, 2008, p.5). Se considera que el "(...) Plan de Equidad se diferencia del anterior Plan de Emergencia porque no se acota temporalmente (se plantea metas progresivas) en razón de que se propone la reconfiguración del sistema de protección social" (CONSEJO NACIONAL DE POLÍTICAS SOCIALES, 2009, p.17).

Recuerda Camou (1997) que la demanda de conocimiento especializado que las reformas estatales sucedidas en América Latina a partir de la segunda posguerra produjeron, entre otros fenómenos, fue la incorporación de especialistas y técnicos egresados de las universidades hacia distintos estratos de la burocracia pública. Es así, que esta tendencia constituye un proceso de largo plazo, no obstante, este proceso de creciente profesionalización de los cuadros burocráticos de la función pública presenta actualmente algunas particularidades que merecen atención respecto a la década de 1980: i- un entramado institucional con cierta "autonomía relativa" formado por fundaciones, centros de investigación, think tanks y consultoras especializadas que elaboran diagnósticos y recomendaciones "(...) que mientras contribuyen a configurar sus propios intereses, también inciden sobre las políticas estatales a fin de disciplinar a los agentes económicos y al propio Estado (...)"; ii- se destaca el carácter semipúblico (o semiprivado) de estas organizaciones, que en general se constituyen como entidades civiles sin fines de 
442

lucro cuyas fuentes de financiación son diversa, empresas, fundaciones internacionales y hasta el propio Estado; iii- la incorporación de técnicos sin credenciales políticas pero con reconocidos antecedentes profesionales a las funciones de asesoramiento en la gestión directa de los asuntos de política pública, "(...) son ellos mismos y por derechos propio parte sustantiva y fundamental de una nueva élite dirigente que desde los albores de las recuperaciones democráticas se han venido abriendo paso en la pirámide del poder" (p.12-14).

En el caso uruguayo Canzani y Cancela (2015) ubican sobre finales del siglo XX la incorporación de los sistemas de información de forma sostenida en el proceso de las políticas públicas a razón de tres aspectos sustantivos; i-el surgimiento de comunidades académico-profesionales con capacidad de producir información y operar sobre los sistemas estadísticos oficiales; ii- la incorporación en el sector público de la perspectiva de "presupuesto por resultados"; y iii-el desarrollo y extensión de Programas de Transferencia de Renta Condicionada (PTRC), ya sea porque cuentan con financiación externa o porque se trata de mecanismos no contributivo, y éstos enfrentan mayores requerimientos de rendición de cuentas.

Esta tendencia de incorporación de sistemas de información a la gestión pública que precedentemente hemos nombrado como tecnificación de la gestión pública en referencia al impulso del paradigma sociotécnico, en Uruguay fue impulsado por los organismos internacionales mediante, entre otros mecanismos, la evaluación externa de proyectos internacionales que señalaban: i- la dificultad de evaluar programas mal formulados y con ausencia de sistemas de gestión de datos; ii- la necesidad de articular las iniciativas ad hoc con las políticas públicas a modo de asegurar su sostenibilidad; y iii- "(...) recomiendan -y en muchos casos imponen- requisitos crecientes para la formulación de nuevas iniciativas, entre las cuales se encuentran mayores exigencias en términos de los sistemas de información" (CANZANI; CANCELA, 2015, p.58-59).

De Armas y Garcé (2004) también reconocen que, si bien en las últimas décadas la incorporación de conocimiento especializado en las políticas públicas en Uruguay se ha incrementado sensiblemente, entienden que los saberes especializados han permanecido bajo el contralor de los políticos profesionales representantes de los partidos políticos de turno. Siendo así, argumentan que en aquellos países en que los actores políticos controlan la incorporación de conocimiento especializado a la gestión pública “(...) no será sencillo encontrar casos claros de transferencia de paradigmas de políticas desde las IFIs. La pauta normal será la resistencia, la autonomía, la heterodoxia" (p.78). En sintonía con este planteo entienden que,

(...) la incidencia real de las orientaciones doctrinarias de las IFIs en la reforma de las políticas también depende de la densidad del conocimiento especializado producido endógenamente. A medida que aumenta la capacidad técnica doméstica, disminuyen las posibilidades de que las agencias internacionales puedan transferir paradigmas de políticas. (DE ARMAS; GARCÉ, 2004, p.79)

Las relaciones entabladas entre Uruguay y los organismos internacionales datan de la década del 60. El país firmó las primeras cartas de acuerdo con el FMI en: setiembre de 1960, junio de 1961, setiembre de 1962, y mayo de 1966. Al respecto Couriel y Lichtensztejn (1967) desde una lectura crítica al proceso de dependencia que se comenzaba a edificar no solo en lo económico, sino en lo cultural, destacan allí el papel de la investigación en ciencias sociales y denuncian que en aquel momento buena parte se encauzaba al “(...) estudio del statu quo sin análisis crítico de las contradicciones de nuestra sociedad (...)", y que por tanto, contribuían directamente a legitimar los intereses de los cuadros burocráticos del gobierno y de las empresas extranjeras (p.171).

Estudio más recientes observan que durante la década del 80 el vínculo entre expertos y políticos en Uruguay experimentó un cambio significativo respecto a décadas pasadas, se verificó una mayor intención de los elencos gobernantes de apoyar sus decisiones en evidencia empírica e investigaciones. No obstante, destacan que durante el gobierno del FA (2005), partido de izquierda que hasta el presente se mantiene en el gobierno, hubo una importante cooperación entre los expertos y la organización de 
gobierno, siendo particularmente significativo en el Ministerio de Economía y Finanzas (MEF), en la Oficina de Planeamiento y Presupuesto (OPP) y en el MIDES. La institución demandada en esta coyuntura política fue la Universidad de la República (UdelaR), con participación destacada de la Facultad de Ciencias Sociales (FCS) y la Facultad de Ciencias Económicas y de la Administración (FCEA). De esta forma, subrayan que siendo el componente técnico (conocimiento especializado) para los países que reciben asistencia una exigencia que los organismos internacionales colocan como estrategia para medir el impacto de las medidas sugeridas e implementadas, en este contexto referido fue uno de los aspectos que fomentó el uso de la investigación social, lo que evidencia el papel decisivo de la participación de los organismos internacionales en los procesos de reforma política (GALLARDO, GARCÉ; RAVECCA, 2010).

Es posible afirmar que si bien el uso político del conocimiento especializado es frecuente en el Uruguay, algunas áreas presentan mayores niveles de requerimiento de conocimiento producto de la investigación y estarían pensando la experticia técnica "en sí misma", siendo un ejemplo de ello el MIDES con la implementación del Plan de Atención a la Emergencia Social (PANES) y posteriormente el Plan de Equidad Social (PES) y la Red de Asistencia e Integración Social (RAIS), para lo cual incorporó (el MIDES) a sus cuadros técnicos investigadores, particularmente de la UdelaR, mediante convenios interinstitucionales de cooperación (GALLARDO, GARCÉ; RAVECCA, 2010).

\section{Conclusiones}

Este esquema exploratorio que trianguló analíticamente la participación de los organismos internacionales en los procesos de elaboración de política pública en los países periféricos mediante la asistencia internacional para el desarrollo y el papel del conocimiento especializado, con observaciones sobre la reforma en el esquema de protección social en Uruguay en los últimos diez años, deja planteado una serie de interrogantes cuyas aproximaciones sucesivas (respuestas) solo pueden ser respondidas en referencia a los procesos socio políticos que caracterizan la región sino que también en la particularidad del caso objeto de estudio.

No obstante, compartimos la observación que realiza Ornellas Mauriel (2009) para la política de Asistencia Social en Brasil en referencia a que la participación de los organismos internacionales es determinante en la configuración de las políticas de combate a la pobreza, que en el caso uruguayo se expresa en la institucionalización del PES y la RAIS, a saber, “(...) expressa mais um conjunto de iniciativas orquestradas internacionalmente pelas organizações econômicas multilaterais do que um grupo de mecanismos pontuais de enfrentamento da questão social concebidos separadamente em escala nacional" (p.64).

A estas observaciones sumamos las advertencias que Martínez Franzoni (2006) realiza y que apuntan a la riguridad metodológica que estos estudios deben atender, para la autora los análisis sobre política pública deben reparar en al menos tres aspectos: i-la especificidad de las transformaciones en las políticas sociales respecto a las políticas macroeconómicas; ii-concebir la relación entre los organismos internacionales y los países como un proceso en el cual intervienen, además de la "condicionalidad" múltiples mecanismos; y iii- considerar la variabilidad en la agenda de las políticas promovidas por los organismos internacionales a lo largo del tiempo, lo cual supone distanciarse de la idea que estas instituciones poseen una agenda única e invariable a lo largo del tiempo.

Finalmente, afirma Bourdieu (1993, p. 2);

(...) las administraciones públicas y sus representantes son grandes productores de "problemas sociales" que la ciencia social no hace a menudo sino ratificar al retomarlos por su cuenta como problemas sociológicos (...) el pensamiento del pensador funcionario está atravesado de cabo a rabo por la representación oficial de lo oficial (...). 
Esta exposición más que certezas pretende compartir nuevos puntos de partida (preguntas) y entradas analíticas a este vasto campo de estudio, a saber, ¿qué rol deberían desempeñar los académicos y las Universidades públicas en la generación de política pública?, ¿qué rol se les adjudica a las ciencias sociales y que rol asumen?, ¿qué contenidos político-reflexivo deberían orientar las producciones académicas?, ¿qué diferencias y similitudes podemos establecer en la participación de los organismos internacionales en los proceso de reforma de cada país y qué papel desempeña el conocimiento especializado?, ¿qué sucede en cada país con otros actores relevantes como los partidos políticos, grupos económicos, corporaciones, en la generación de política pública?

\section{Referencias}

ÁLVAREZ LEGUIZAMÓN, S. Gubernamentalidad Neoliberal y Focopolítica en América Latina: Los Programas de Transferencias Condicionadas ¿Políticas de Cohesión Social? En BARBA, N. y COHEN, N (Org.) Perspectiva Críticas sobre la Cohesión Social. Buenos Aires: Consejo Latinoamericano de Ciencias Sociales (CLASCO). 2011, p.251-285.

. Los cambios operados en las concepciones de gestión de programas sociales a partir del financiamiento internacional. En: VI Congreso Internacional del CLAD sobre la Reforma del Estado y de la Administración Pública Buenos Aires, Argentina. Panel: La evaluación y gestión de las políticas sociales en las últimas dos décadas: problemas y perspectivas. 2001

BORÓN, A. Las ciencias sociales en la era neoliberal: entre la academia y el pensamiento crítico. Centro de Estudios Latinoamericanos Justo Arosemena. 2006. Disponible en: <http://bibliotecavirtual.clacso.org.ar/ar/libros/panama/cela/tareas/ tar122/03boron.pdf.

BOURDIEU, P. Espíritus de Estado. Génesis y estructura del campo burocrático. Artículo originalmente publicado en Actes de la Recherche en Sciences Sociales, n. 96-97. 1993. Disponible en: http://pierre-bourdieu.blogspot.com.uy/2006/07/gnesis-yestructura-del-campo.html

CAMOU, A. Los consejeros del príncipe. Saber técnico y política en los procesos de reforma económica en América Latina. Revista Nueva Sociedad, n.152, p. 54-67. 1997.

CANZANI, A; CANCELA V. Una mirada al Sistema de Información del MIDES: contexto, desarrollo, estructura, valoración y posibilidades. Uruguay: Cuadernos de Ciencias Sociales y Políticas Sociales-Facultad de Ciencias Sociales, UdelaR. 2015. ISSN: 23936266.

CARDOSO DA MOTTA V. Da ideologia do capital humano à ideologia do capital social: as políticas de desenvolvimento do milênio e os novos mecanismos hegemônicos de educar o conformismo. Tesis doctorado. Rio de Janeiro: Universidade Federal do Rio de Janeiro-Programa de Pós-Graduação em Serviço Social, 2007.

CASTEL, R. La inseguridad social. ¿Qué es estar protegido? Buenos Aires, Argentina: Manantial, 2004.

COURIEL, A.; LICHTENSZTEJN, S. EI FMI y la crisis nacional. Uruguay: Biblioteca de Cultura Universitaria, 1967.

CORBALÁN, M. América Latina frente a los organismos internacionales de crédito. Intervención y disciplinamiento. Función política de los organismos internacionales de crédito. Ciclos, Año XIV, v. XIV, n. 27, 2004. Disponible en: http://bibliotecadigital.econ.uba.ar/download/ciclos/ciclos_v14_n27_01.pdf.

DE ARMAS, G.; GARCÉ A. Política y conocimiento especializado: la reforma educativa en Uruguay (1995-1999). Revista Uruguaya de Ciencia Política, n. 14, Montevideo: Instituto de Ciencia política, 2004.

FRERES, Ch.; MARTÍNEZ, I.; ANGULO, O. La inserción de América Latina en el cambiante sistema internacional de cooperación. España: Fundación Carolina-CeALCI. Documento de Trabajo n. 46, 2010. Disponible en: https://www.fundacioncarolina.es/wpcontent/uploads/2014/ 08/DT46.pdf

FRERES Ch. Reflexión acerca de la Arquitectura Internacional de la Ayuda. En FRERES, Ch., MARTínEZ, I.; ANGULO, O. (Org.), La inserción de América Latina en el cambiante sistema internacional de cooperación. España: Fundación Carolina-CeALCl. Documento de Trabajo n. 46, 2010. Disponible en: https://www.fundacioncarolina.es/wp-content/uploads/2014 / 08/DT46.pdf

GALLARDO, J; GARCÉ, A.; RAVECCA, P. De la coyutura a la estructura: uso de la invewstigación social en las políticas públicas de Uruguay durante el gobierno de Tabaré Vazquéz (2005-2009). En M. MANCEBO, E. y NARBONDO, P. (Org.) Reformas del Estado y políticas públicas de la Administración Vázquez: acumulaciones, conflictos y desafíos. Uruguay: CLASCO, Instituto de Ciencia Política-UdelaR, 2009.

MARTÍNEZ FRANZONI. ¿Presión o legitimación? Poder y alternativas en el diseño y adopción de la reforma de salud en Costa Rica, 1988-1998. Revista História, Ciências, Saúde - Manguinhos, Brasil, v. 13, n. 3, p. 591-622, 2006.

MATO, D (2007) Think tanks, fundaciones y profesionales en la promoción de ideas (neo)liberales en América Latina. En: GRIMSON, A. (Org.) Cultura y neoliberalismo. Buenos Aires: Consejo Latinoamericano de Ciencias Sociales CLACSO, p. 19-42, 2007. 
MIDAGLIA, C. Entre la tradición, la modernización ingenua y los intentos de refundar la casa: la reforma social en el Uruguay de las últimas tres décadas. En BARBA, C. (Orgs.). Retos para la Integración Social de los Pobres en América Latina (80-105). Buenos Aires: Consejo Latinoamericano de Ciencias Sociales (CLASCO), p. 80-105, 2008.

MONTÚFAR, C. Hacia un nuevo marco interpretativo de la asistencia internacional para el desarrollo. En GÓMEZ J. (Org.). América Latina y el (des) orden global neoliberal. Hegemonía, contrahegemonía, perspectivas. Buenos Aires: Consejo Latinoamericano de Ciencias Sociales (CLASCO), p.245-258, 2004.

MURILLO, S. Colonizar el dolor. La interpelación ideológica del Banco Mundial en América Latina. El caso argentino desde Blumberg a Cromañón. Buenos Aires: Consejo Latinoamericano de Ciencias Sociales CLACSO, 2008. ISBN 978-987-1183-90-6. Disponible en: http://bibliotecavirtual.clacso.org.ar/ar/libros/becas/murillo/00Intro.pdf

ORNELLAS MAURIEL A. Relações internacionais, política social e combate à pobreza. Revista da Faculdade de Serviço Social da Universidade do Estado do Rio de Janeiro, v 6, n. 23, p. 43-67, 2009.

ORNELLAS MAURIEL A. Combate à pobreza e desenvolvimento humano: impasses teóricos na construção da política social na atualidade. Tese (Doutorado) - Departamento de Ciência Política do Instituto de Filosofia e Ciências Humanas, Universidade Estadual de Campinas, São Paulo, 2008.

PLOTKIN, M.; ZIMMERMANN, E. Los saberes del Estado. Buenos Aires: Edhasa, 2011.

STEIN, R. Capital social, desarrollo y políticas públicas en la realidad latinoamericana. Brasil: Departamento de Trabajo Social, Universidad de Brasília. Unidad de Políticas Comparadas (CSIC). 2003. Disponible en: http://digital.csic.es/bitstream/10261/1567/1/dt-0305.pdf?origin =publication detail. Revisado 2 de mayo 2013.

\section{Fuentes documentales}

AGENCIA URUGUAYA DE COOPERACIÓN INTERNACIONAL (AUCI). Los países Latinoamericanos en el escenario de la cooperación internacional. 2011. Disponible en: www.auci.gub.uy

CONSEJO NACIONAL DE POLÍTICAS SOCIALES (CNPS) Balance y Perspectivas 2005-2009. Uruguay: CNPS-Presidencia de la República. 2009.

CONSEJO NACIONAL DE POLÍTICAS SOCIALES (CNPS). Plan de Equidad. Uruguay: Presidencia de la República. 2008.

\footnotetext{
${ }^{1}$ En la reconfiguración del nuevo orden mundial, Estados Unidos (EEUU) surge como potencia y su moneda, el dólar, reemplaza al patrón oro como moneda internacional de intercambio, además en esa coyuntura la idea de contener la expansión comunista se extiende rápidamente. El Fondo Monetario Internacional (FMI) nace con la conferencia de Bretton Woods celebrada en 1944 en EEUU y comienza a funcionar en 1947, el Banco Mundial (BM) que primero fue denominado Banco de Reconstrucción y Fomento (BIRF), "mellizo" del FMI nace con perfil más bajo y financiado con recursos exclusivos de EEUU (COURIEL; LICHTENSZTEJN, 1967). ${ }^{2}$ La distinción entre organismos multilaterales $u$ organismos internacionales radica en la postura teórico-ideológica que asumen las producciones en general, aunque no debe tomarse de forma taxativa ya que no siempre es explícita esta distinción. Usualmente aquellas lecturas más críticas en torno a la asistencia de estos organismos se refieren a organismos internacionales o Instituciones Financieras Internacionales (IFIs), en tanto posturas más propositivas refieren a organismos multilaterales, agencias de desarrollo, agencias cooperación. Este análisis asumirá la segunda denominación, organismos internacionales.

${ }^{3}$ Las expresiones en cursiva con propias del autor.

${ }^{4}$ El paradigma sociotécnico siendo impulsado y legitimado por los organismos internacionales es un modelo de gestión pública que se inspira en una "filosofía empresarial" de las políticas públicas en general, y de las sociales en particular, y que se sustenta en la idea de "gerenciamiento" para la búsqueda de eficiencia y eficacia en la ejecución de las políticas sociales. Esta modalidad se distancia del que había primado en los modelos de gestión precedentes y conduce a una tendencia en la que coexisten dos lógicas en la ejecución de los programas, la político/clientelar y la lógica técnico/eficientista (ÁLVAREZ LEGUIZAMÓN, 2001, p.8).

${ }^{5}$ La "teoría del capital humano" tiene origen en estudios elaborados bajo la influencia de Theodore Schultz (desde 1950), publicados en los años 1960 en su libro "Capital Humano", cuya repercusión e impacto dentro del área económica lo llevará a obtener el Premio Nobel de Economía en 1968. Estos trabajos procuran demostrar que la educación se traduce en términos de inversión productiva, es decir, que la educación y la formación constituyen instrumentos decisivos para el desempeño productivo de los individuos. (STEIN, 2003; CARDOSO DA MOTTA, 2007)

${ }^{6}$ Entendemos por marcos cognitivos de referencia aquellas definiciones y operaciones (teórico- metodológicas) que fundamentan la política socio-asistencial. En otras palabras, la caracterización y explicación del problema en torno a la pobreza, la caracterización de la población, las propuestas de abordaje y los instrumentos y mecanismos que se sirven para el diseño, implementación y evaluación constituyen soportes que fundamentan y sostienen la reingeniería de esta política.
} 